\title{
RADIATIVE RECOMBINATION AND OTHER PROCESSES RELATED TO EXCESS CHARGE CARRIERS, DECISIVE FOR EFFICIENT PERFORMANCE OF ELECTRONIC DEVICES
}

\author{
A. Wincukiewicz, W. Mech, S. Grankowska, A. Wolos, A. Drabinska, T. Slupinski, \\ K.P. Korona, and M. Kaminska \\ Institute of Experimental Physics, Faculty of Physics, University of Warsaw, Pasteura 5, 02-093 Warsaw, Poland \\ Email: maria.kaminska@fuw.edu.pl
}

Received 19 February 2018; accepted 22 March 2018

\begin{abstract}
We present selected semiconductor (inorganic and organic) structures for which non-radiative recombination of excess charge carriers is very high, luminescence suppressed and its lifetime substantially shortened. Processes competitive with radiative energy emission are discussed. The importance of Shockley-Read-Hall recombination in materials with high impurity or defect concentration, applied in ultrafast devices, is shown. Also, charge transfer process in solar cells is discussed in the context of luminescence quenching of individual components of an active layer.

A part of the shown research was a subject of our common work with Prof. Arūnas Krotkus during the period from 1994 till 2006.
\end{abstract}

Keywords: radiative and non-radiative recombination, charge transfer, time-resolved luminescence

PACS: 78.47, 78.66.Fd, 78.66

\section{Introduction}

Excitation of semiconductors with electromagnetic radiation of energy above the band-gap (or HOMO-LUMO distance, in the case of organic semiconductors) leads to creation of electronhole pairs which disturbs the system from its thermal equilibrium. Also, electrical injection of carriers results in the non-thermal-equilibrium state. Any disturbance of the thermal equilibrium condition triggers relaxation and recombination processes which restore the system equilibrium. In general, the recombination processes can be classified into radiative (involving photons) and non-radiative processes.

When the competition of non-radiative processes is high, the radiative recombination is strongly diminished and the luminescence lifetime is substantially shortened. However, the ef- ficient radiative recombination is a desirable feature of many devices, especially those which are light sources or photodetectors. In light emitting diodes the intensity of spontaneous emission of light is optimized. For lasers, the steady state excited population, decisive for the emitted power in a form of stimulated emission, is governed by the lifetime of carriers at the excited state. In detectors based on photocurrent, the sensitivity depends also on the photo-excited carrier lifetime. Besides light sources and photodetectors, low non-radiative recombination is also important for materials which are components of an active layer in solar cells. A long lifetime of photo-excited carriers results in efficient charge transfer, the most important process that determines efficiency of solar cells.

On the other hand, fast recombination of excess carriers is an absolutely necessary attribute 
of ultrafast devices, like picosecond switches, fast detectors, saturable absorber mirrors or terahertz radiation emitters. The reason for lifetime shortening of photo-excited carriers is mostly nonradiative channels of recombination in which defects play a leading role.

In the following paragraphs, we will describe recombination mechanisms that determine the recombination rate and decide on the efficient operation of different devices. We will also consider charge transfer in solar cell structures and its influence on the radiative recombination of active layer components. Then, we will refer to these processes in the case of two selected semiconductor structures: doped/defected semiconductors and active layers in organic photovoltaics based on polymers or perovskites. The presented results of time-resolved luminescence were obtained using a Hamamatsu streak-camera with 2.5 ps resolution. The samples were excited by the third harmonic of the pulsed Ti:sapphire laser working at $80 \mathrm{MHz}$. Electron paramagnetic resonance was measured using a Bruker ELEXSYS E580 X-band spectrometer $(9.5 \mathrm{GHz})$ equipped with an Oxford continuous-flow cryostat.

\section{Radiative recombination versus non-radiative processes}

In radiative recombination energy is released in a form of emitted photons. Regarding semiconductor devices, in most cases we deal with bandgap-range radiative recombination, and since a photon carries only a little momentum in comparison with a Brillouin zone size, such radiative recombination is significant only in direct bandgap semiconductors.

The origin of bandgap-range radiation is mainly related to the recombination of excitons (Coulomb bound electron-hole pairs), although free exciton recombination usually occurs at very low temperatures only, whereas at room temperature excitons are ionized. However, in organic semiconductors excitonic recombination is important also at room temperature. A small dielectric constant of such materials results in a strong electrostatic attraction, and thermal energy at room temperature is too low to break down bound electron-hole pairs. Also, in low dimensional structures excitonic recombination at room tem- perature is observed due to localization of charge carriers and therefore a stronger electron-hole attraction force than in bulk materials. In bulk materials the band-gap range luminescence is often dominated by an exciton bound to shallow impurity since the binding localizes the exciton in real space. The other often present luminescence in this energy range is due to the so-called shallow donor - shallow acceptor pairs, and in this case an excited electron at the donor centre recombines with the hole at the acceptor and the process is enhanced by the overlap of electron and hole wavefunctions. Luminescence connected with deep impurities, specifically due to internal transitions within the impurities of rare earths or transition metals, is sometimes exploited in devices as well, especially in solid-state lasers and in luminophore applications.

Radiative recombination is in the first order of a dipole character, and the characteristic radiative recombination time of point charge in vacuum which vibrates harmonically with the frequency $v$ can be estimated from the formula [1]

$$
\tau=\frac{3 m c^{3}}{8 \pi^{2} e^{2} v^{2}} .
$$

Assuming wavelength from the visible range, $\lambda \approx 600 \mathrm{~nm}$, the radiative recombination time $\tau \approx 1.6 \times 10^{-8} \mathrm{~s}$.

Lifetime corresponding to the electron-hole pair radiative recombination in several semiconductors has been calculated theoretically and its values differ from the one given above. This lifetime changes very much with the energy gap of a semiconductor, being as short as $0.6 \times 10^{-6} \mathrm{~s}$ for InSb (0.17 eV energy gap), and as long as $500 \mathrm{~s}$ for $\mathrm{GaAs}$ (1.4 eV energy gap) [2, 3]. However, the experimental values of luminescence lifetime in the corresponding semiconductors never reach these theoretical numbers.

The luminescence lifetime of the order of nanoseconds is typical of high quality crystals, like GaAs or InP. As one can see, even in such semiconductors of a good crystalline structure, the recombination time is already strongly reduced relative to the theoretical values of radiative recombination lifetimes. This is due to omnipresent defects and impurities (even nonintentional) which trigger nonradiative processes discussed below. The recombination time can be further 
substantially reduced in semiconductors with a high concentration of defects or impurities.

On the other hand, in organic or hybrid semiconductor structures for photovoltaics the radiative recombination time is also reduced, but because of a quite different mechanism. The socalled charge transfer process is active in such structures, relying on efficient separation of electrons and holes into different materials of an active layer, and leading to a decrease of their luminescence intensity and its faster decay.

\subsection{Non-radiative recombination in semiconductors}

Non-radiative dissipation of energy occurs as a result of the crystalline defect and impurity presence, or Auger processes. The energy released in non-radiative processes usually leads to the phonon excitation and the increase of local temperature. As a result, not only the decrease of radiative emission intensity and shortening of its lifetime takes place, but also the degradation of electronic devices may occur.

In the Auger recombination the excited electron-hole pair gives its energy to a third charge carrier exciting it to higher energy level within its energy band. Afterwards, the third carrier typically loses its energy to thermal vibrations. The Auger recombination is significant only in materials with a high density of carriers. Its probability (increasing with carrier density) is typically low because it involves a three-particle interaction. Also, the third particle must start the process being in a high energy state - a partially empty band. Therefore, the Auger recombination can be expected to be significant only in highly doped materials or under a strong optical or electrical injection of carriers, and will not be discussed further.

When band carriers are trapped by deep defects, they become localized, and often release their energy through phonon emission because of a usually strong electron-phonon coupling at such centres. In some cases the radiative recombination at defect states takes place, but its energy differs from that of the band gap and is characteristic of defect energy levels. The most important defects in semiconductors include point defects (vacancies, interstitials, antisites and their complexes), dislocations, stacking faults, and grain boundaries. All of them strongly interact with the crystalline lattice.
Lattice vibration occurs at a frequency of the order of $10^{12}-10^{13} \mathrm{~s}^{-1}$, so the typical time of energy dissipation by lattice vibration is of the order of picoseconds. Such defect-assisted recombination is called the Shockley-Read-Hall (SRH) recombination and constitutes a dominant recombination process in indirect bandgap materials, like silicon, where radiative processes are not efficient. It can also dominate in direct bandgap materials with defects of high density and compensating shallow centres, and therefore when free carrier concentration is low. The SRH recombination rate is proportional to the excess carrier density beyond the thermal equilibrium value $\Delta n$, defect density $N_{T}$ and defect capture cross-section $\sigma$. The higher the SRH recombination rate, the shorter the excess carrier lifetime which can be calculated from the formula

$$
\frac{1}{\tau_{\mathrm{nr}}}=N_{T} \sigma v,
$$

where $v$ is the thermal velocity of carriers. Therefore, the higher the concentration of defect centres, the shorter the relaxation time of excess carriers.

\subsection{Charge transfer in solar cells}

Charge transfer is a crucial process, decisive of efficient work of organic or hybrid solar cells. It consists in transfer of one kind of electrical charge (electrons) from the LUMO level of one component (often called a donor) to the LUMO level of the other component (often called an acceptor) of the active layer of an organic or hybrid solar cell. Simultaneously holes, if excited in the acceptor material to its HOMO level, are transferred in the opposite direction. The charge transfer process has been a subject of intensive studies in the recent years [4-6]. In Fig. 1 the working principle of a polymer solar cell is presented indicating all important stages, and one of the processes shown is the charge transfer. In order for charge transfer to happen the donor material must have higher HOMO and LUMO energy levels than the corresponding levels of the acceptor material, because the process occurs due to the energy gain of the transferred charges.

Organic solar cells are a subject of interest because they provide hope to satisfy the emerging 


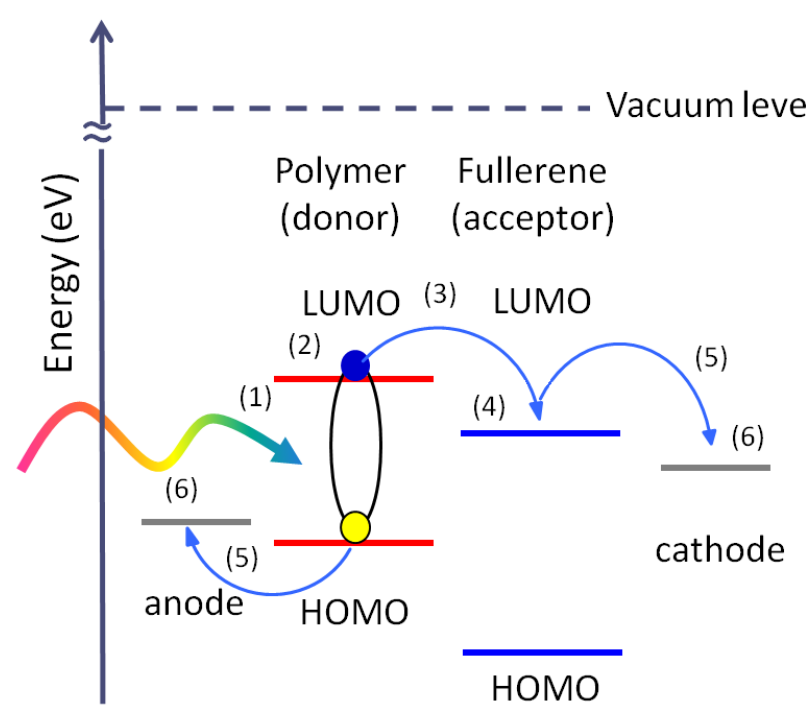

need for sustainable energy via application of simple, cheap and environment-friendly technologies. In the last few years high expectations are connected with cells based on hybrid perovskites, for which the efficiency has been highly improved in a relatively short time of research [7]. The materials used for organic photovoltaics should have an intensive excitonic luminescence of a long lifetime, what proves that the number of defects or impurities interacting with excitons is small. Therefore, in such materials exciton diffusion (or in the case of perovskites free charge carrier diffusion, because the exciton binding energy is low [8]) to the interface between the components of an active layer, and charge separation will not be interrupted by non-radiative recombination processes.

\section{Semiconductors with high concentration of deep impurities or defects}

Deep impurities or defects present in semiconductors at a high enough concentration (so they can compensate other impurities or defects) cause Fermi level pinning at their energy level. Such materials have a low free carrier concentration, and electrical transport is dominated by hopping within the defect band at least at low temperatures [ 9 , 10], with this range increase to higher temperatures when the defect concentration increases. It
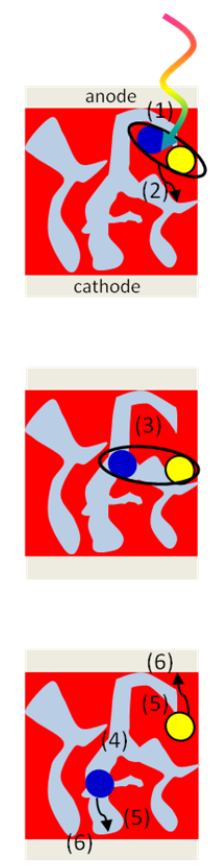

Fig. 1. Working principle of polymer organic solar cells with an active layer in the form of a bulk heterostructure consisting of polymer and fullerene components. On the left: simplified energy diagram; on the right: schematic electron and hole behaviour at different stages (1-6) taking place. (1) photon absorption and formation of the electron-hole pair (exciton),

(2) exciton diffusion to the donor/ acceptor interface, (3) exciton dissociation at the interface through charge transfer, (4) separation of the still Coulomb bound electron-hole pair, (5) migration of the hole (in the donor) and the electron (in the acceptor) towards the respective electrodes and (6) charge collection.

is why materials with a high defect concentration strongly change their conductivity under illumination. At the same time, because of the efficient SRH recombination, the lifetime of photo-excited carriers is short. All these features are beneficial for applications of materials with a high defect concentration - both as insulating layers as well as active components of ultra-fast devices.

Doping with transition metals was at the beginning the most common way to achieve semiinsulating properties of semiconductor materials - doping of GaAs with chromium can serve here as a leading example. Transition metals in the crystal lattice of II-VI and III-V semiconductor compounds substitute for cation and their electrons from the not-fully filled d orbit are well localized. Chromium in GaAs [11] has a deep acceptor level at the middle of the energy gap, can compensate net silicon shallow donors and makes GaAs highly resistive. However, doping at high concentration is often difficult and limited by dopant solubility. Also, some dopants like chromium are not stable at temperature of device processing or operation, and their diffusion leads to degradation. Much more stable than chromium is the native point defect of bulk GaAs, called EL2 [12]. Two experiments allowed one to determine its nature as isolated arsenic antisite. The first one was electron paramagnetic resonance (EPR) of this defect, which showed the characteristic four-line 
spectrum related to the $\mathrm{As}_{\mathrm{Ga}}^{+}$charge state (a single electron spin of $1 / 2$ interacting with the nucleus spin of $3 / 2$ of the parent As atom) - see Fig. 2(a) [13]. The other one was the optical absorption connected with the EL2 internal transition (see Fig. 2(b)) which revealed splitting of the zero phonon line under uniaxial pressure, characteristic of the substitutional $\mathrm{As}_{\mathrm{G}}^{0}$ centre in the zinc blend lattice in $T_{d}$ symmetry [14, 15. It was found that a typical concentration of the arsenic antisite defect in bulk GaAs, of the order of $10^{16} \mathrm{~cm}^{-3}$, can be substantially increased by means of molecular beam epitaxy (MBE) growth of GaAs under nonstoichiometric As-rich conditions (arsine overpressure) at lower than usual substrate temperature [16, 17]. The low temperature MBE growth (LTG) of GaAs in a temperature range of $180-250^{\circ} \mathrm{C}$ resulted in the concentration of arsenic antisites decreasing from a few times $10^{20} \mathrm{~cm}^{-3}$ down to a few times $10^{17} \mathrm{~cm}^{-3}$ with the growth temperature increase [10]. The arsenic antisite defect acts as a deep donor and its $\mathrm{As}_{\mathrm{Ga}}^{0} / \mathrm{As}_{\mathrm{Ga}}^{+}$energy level is located near the GaAs mid-gap. It keeps the Fermi level deeply in the bandgap by compensation of net acceptors (shallow carbon acceptors and/or deep triple acceptors of gallium vacancies). The high concentration of the arsenic antisite defects is evidently above that of the thermal equilibrium in LTG GaAs and results from the low temperature of the growth process, which suppresses defect mobility within the GaAs lattice. Post-growth an- nealing at temperatures in a range of $400-650^{\circ} \mathrm{C}$ is typically applied and by this means the crystalline quality and charge carrier mobility of LTG GaAs can be notably improved [10]. The annealed material still keeps a high arsenic antisite defect concentration, of the order of $10^{18} \mathrm{~cm}^{-3}$, although a substantial part of arsenic antisite defects forms arsenic precipitates during annealing [18].

Below, among others, we refer to two examples of substantial shortening of the excess carrier lifetime in semiconductors, namely to InP:Mn and LTG GaAs. These materials were a subject of our common studies with prof. Arūnas Krotkus from the Semiconductor Physics Institute in Vilnius, Lithuania, who in the last twenty years has performed the prominent research of a broad spectrum of semiconductor materials with a short excess carrier lifetime and showed their high usefulness in ultrafast optoelectronic devices, like $\mathrm{THz}$ emitters or $\mathrm{THz}$ detectors (see [19-29] for some of the most prominent results of A. Krotkus).

\section{Fast carrier recombination in semiconductors with high impurity or defect concentration}

Typical luminescence lifetimes observed in good crystalline quality bulk GaAs or InP in the energy region of their band gap are in the range of nanoseconds. Luminescence lifetime can be decreased by doping with deep impurities. Figure 3 presents the timeresolved luminescence of the $\operatorname{In}_{0.4}\left(\mathrm{Al}_{0.75} \mathrm{Ga}_{0.25}\right)_{0.6} \mathrm{As}$ (a)

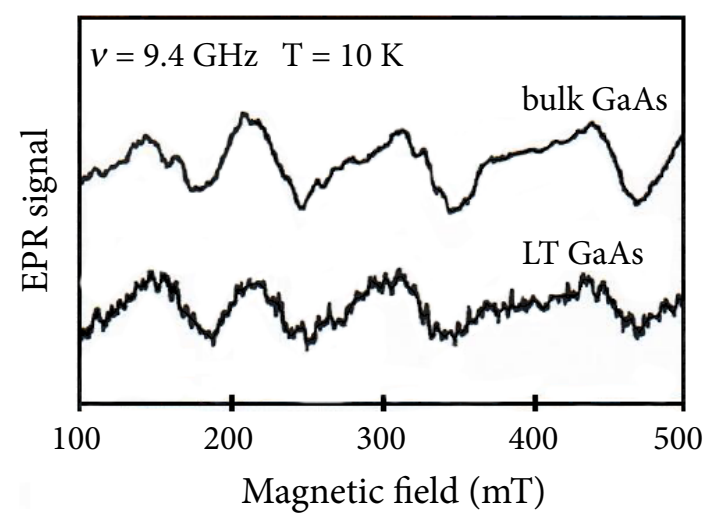

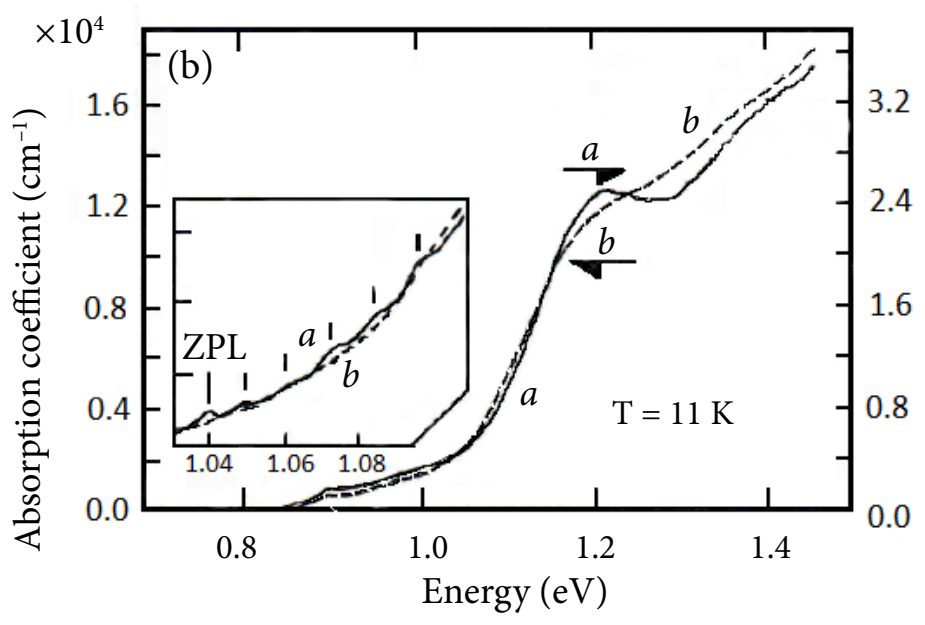

Fig. 2. (a) EPR spectra of bulk and LTG GaAs showing a characteristic quadruplet of the arsenic antisite defect in the singly ionized charge state, and (b) near-infrared absorption of the arsenic antisite defect in the neutral charge state of bulk GaAs, continuous curve $a$, and LTG GaAs, dashed curve $b$ (note the $10^{4}$ times higher values of the absorption coefficient for LTG GaAs than for bulk GaAs). 


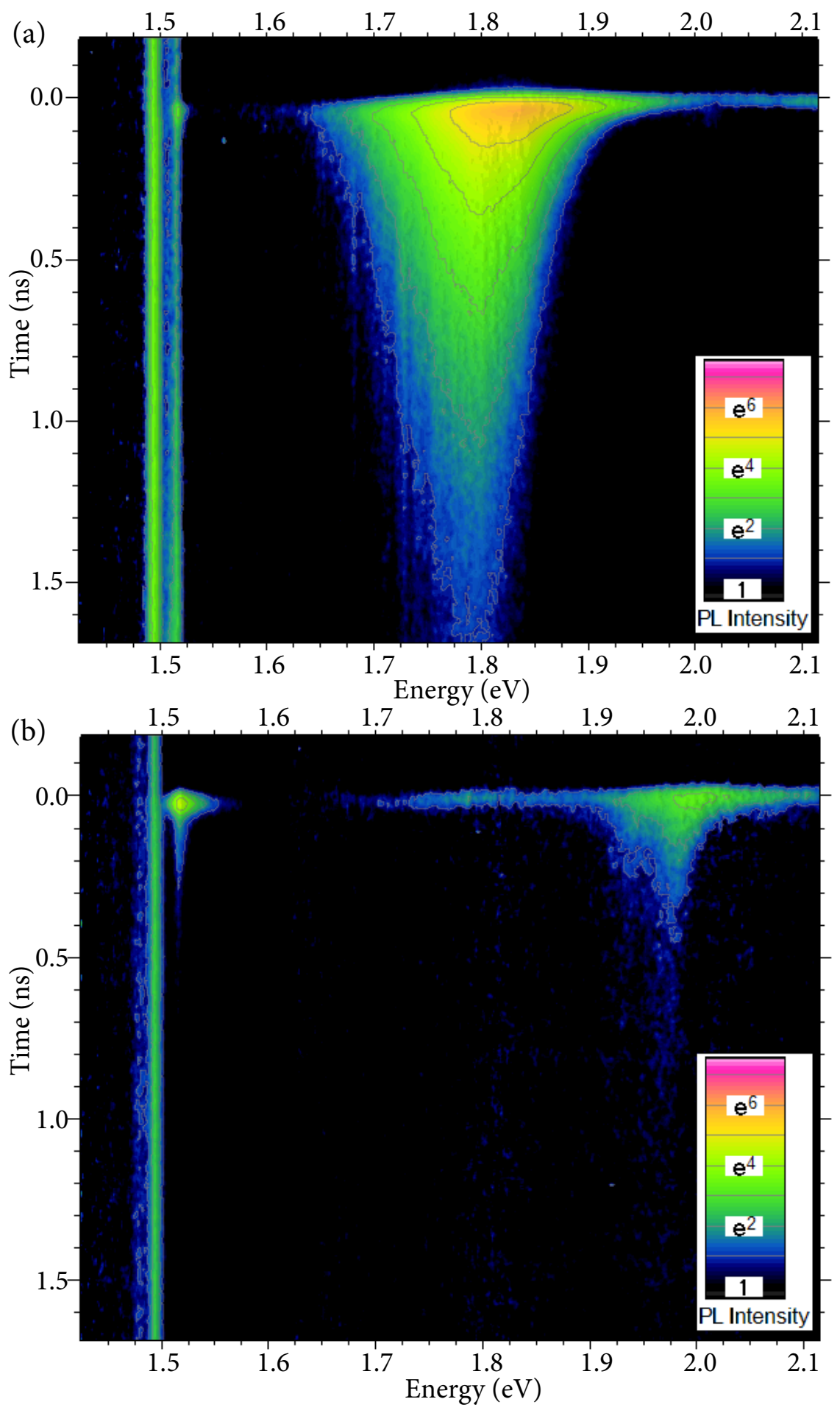

Fig. 3. Time-resolved luminescence of $\operatorname{In}_{0.4}\left(\mathrm{Al}_{0.75} \mathrm{Ga}_{0.25}\right)_{0.6} \mathrm{As}$ quantum dots immersed in $\mathrm{Al}_{0.75} \mathrm{Ga}_{0.25} \mathrm{As}$, grown on $\mathrm{GaAs}$ substrate: (a) undoped quantum dots, (b) quantum dots doped with manganese.

quantum dot structure grown on the GaAs substrate for undoped quantum dots in Fig. 3(a), and quantum dots doped with manganese in Fig. 3(b). The two bright luminescence bands at about $1.5 \mathrm{eV}$ related to the donor-acceptor and the exciton bound to donor (DBE) recombinations in the GaAs substrate are characterized by a long lifetime, in the range of nanoseconds. Such results prove a high quality of the substrate material and its negligible defect concentration. Luminescence of quantum dots is present in the energy range of $1.6-2.2 \mathrm{eV}$. The difference in luminescence intensity and its lifetime, which can be seen by comparing the spectra in Fig. $3(\mathrm{a}, \mathrm{b})$, is obvious, and the luminescence quenching and its lifetime shortening in the Mn doped structure (Fig. 3(b)) can be attributed to the recombination induced by a deep manganese impurity. The luminescence of the GaAs substrate in Fig. 3(b) is also changed in comparison with that in Fig. 3(a), most probably 
due to the Mn diffusion during the growth of layer with dots.

In our common work with A. Krotkus on InP doped with manganese [30], it was shown that manganese centres cause the subpicosecond relaxation of excitons and excess holes. This time was much shorter in comparison with $\mathrm{Mn}$ undoped InP. The neutral donor bound exciton in the n-type InP undoped with $\mathrm{Mn}$ had a lifetime of about a nanosecond. Detailed studies performed by means of time-resolved luminescence, electron transport and electron paramagnetic resonance allowed us to model Mn impurity in InP and understand its role in efficient shortening of the excess carrier recombination time. The recombination was explained by the capture of holes on the $\mathrm{Mn}$ acceptor. The obtained value of the Mn capture cross-section of holes $\sigma$ was found to be of the order of $10^{-12} \mathrm{~cm}^{2}$, which is unexpectedly high for the localized, deep centre. This oddity was explained by assuming trapping a hole by a Mn centre into weakly localized excited states. In the proposed model the neutral $\mathrm{Mn}$ acceptor is formed by a weakly localized hole coupled to the $3 \mathrm{~d}^{5}$ core and the centre cannot be described by localized $3 \mathrm{~d}^{4}$ configuration. On the other hand, the hydrogen model (effective mass theory) of a hole bound on the Mn centre is also not adequate to describe the Mn acceptor in InP. The studies revealed that the neutral Mn acceptor has a relatively well localized ground state with an effective radius of a coupled hole of about $0.7 \mathrm{~nm}$, whereas excited states of this hole are weakly localized with an effective radius of more than $2 \mathrm{~nm}$. The trapping of carriers involves excited states, and therefore the capture cross-section is high.

In Fig. 4 time-resolved luminescence spectra of InP:Mn $\left(7 \times 10^{18} \mathrm{~cm}^{-3}\right.$ of Mn concentration $)$ in the near bang gap region are presented. The spectra show the shallow donor to the shallow acceptor (D-A) luminescence, luminescence of bound exciton $\left(\mathrm{A}^{\circ} \mathrm{X}\right)$, and a high energy tail due to electrons recombining on the $\mathrm{Mn}$ acceptor, decaying very fast in time. The tail is broad because of the high kinetic energy of the recombining electrons which have not enough time to lose their kinetic energy before being trapped. The effective temperature of these electrons estimated from the time resolved luminescence was about $300 \mathrm{~K}$ although the photoluminescence measurement was performed at $7 \mathrm{~K}$.

In InP:Mn with the manganese concentration above $10^{18} \mathrm{~cm}^{-3}$, an increasing contribution of hopping conductivity to the total electron transport with an increase of the Mn content was observed. The hopping conductivity was related to the transport within the Mn defect band which pinned the Fermi level at about $0.2 \mathrm{eV}$ above the valence

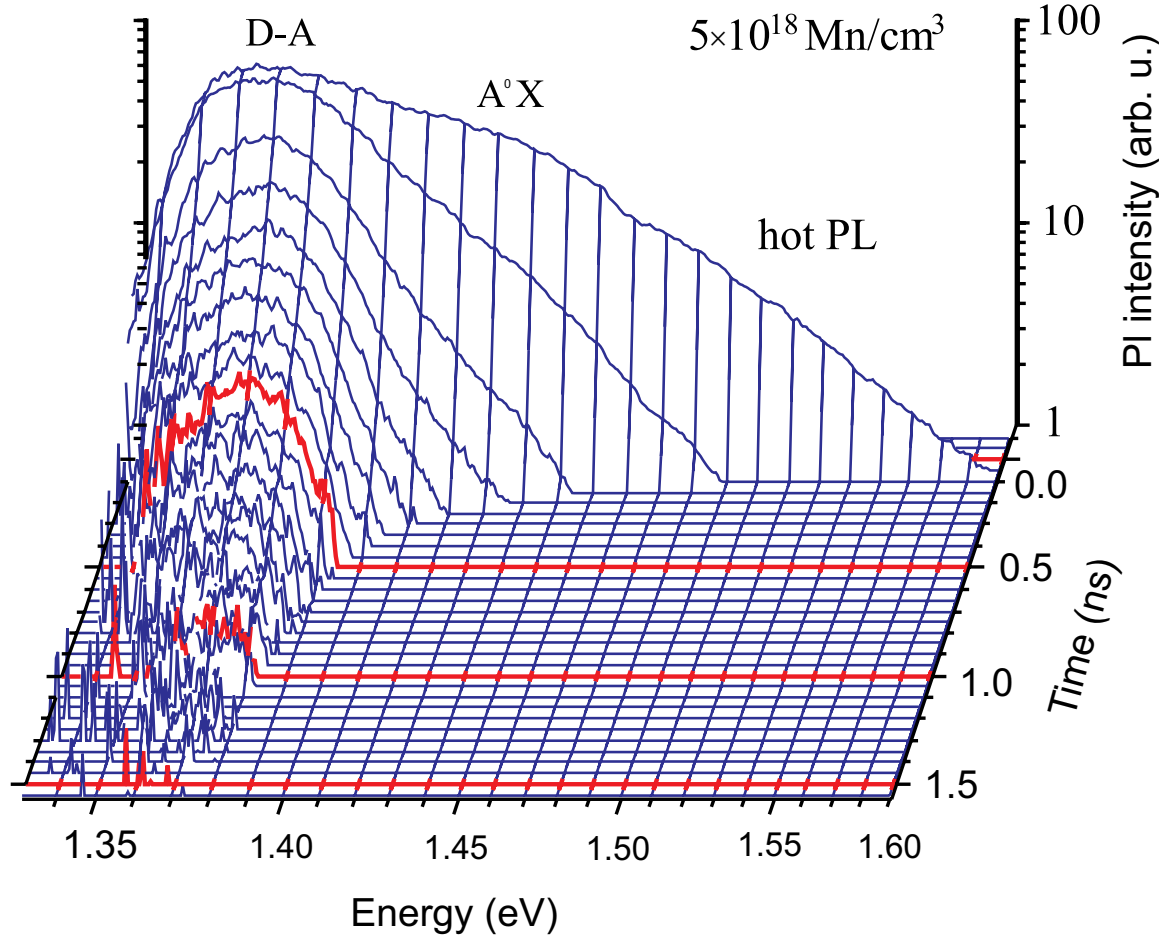

Fig. 4. Time-resolved luminescence measured at $7 \mathrm{~K}$ of InP:Mn in the near-band-gap region. Mn concentration was $7 \times 10^{18} \mathrm{~cm}^{-3}$. 
band, and the width of the band was determined as about $20 \mathrm{meV}$. The defect band corresponded to the $\mathrm{Mn}^{0} / \mathrm{Mn}^{-}$energy level of manganese impurity. The analysis of hopping data for InP with different Mn content allowed us to calculate the wavefunction radius of $0.7 \mathrm{~nm}$ for the $\mathrm{Mn}$-bound hole, as mentioned above.

Another example of the defect governing the lifetime of excess carriers is GaAs, in which the arsenic antisite defect with its mid-gap position creates an effective recombination centre. Especially in LTG GaAs, where the concentration of this defect is particularly high, it clearly activates the SRH recombination mechanism, leading to efficient shortening of the photo-excited carrier lifetime [31, 32]. The effect is so pronounced that LTG GaAs is the most important material for applications in ultrafast optoelectronics [33-35].

Figure 2 presents characteristic EPR and optical absorption spectra of the arsenic antisite defect in bulk GaAs and LTG GaAs grown by the MBE method at $200^{\circ} \mathrm{C}$ [10]. The lack of the fine structure in optical absorption at about $1 \mathrm{eV}$ energy and broadening of the central band with the maximum at about $1.2 \mathrm{eV}$ for LTG GaAs was related to strain present in this material. The concentration of the arsenic antisite defects in such LTG GaAs $\left(200^{\circ} \mathrm{C}\right.$ growth temperature) was about $10^{20} \mathrm{~cm}^{-3}$. This caused a substantial increase of the lattice parameter: a relative difference between the LTG epilayer and the bulk GaAs lattice parameter was found to be about $0.1 \%$.

Electrical transport was dominated by hopping within a defect band of arsenic antisites and the hopping conductivity was already seen at room temperature [10]. A wavefunction radius of carriers trapped at the arsenic antisite defect in LTG GaAs was calculated from the hopping data and the value between $0.5-0.8 \mathrm{~nm}$ was obtained, what indicated a local character of the trap [10, 36].

A high concentration of the arsenic antisite defects in LTG GaAs resulted in ultrafast trapping of electrons with the characteristic time even shorter than 100 fs [37]. Also, LTG GaAs post-growth annealed at temperatures in a range of $600-800^{\circ} \mathrm{C}$ still showed the subpicosecond photo-excited electron decay (electron trapping time was between 0.21 ps) [28, 29]. Several experimental techniques consistently proved this fast carrier dynamics in LTG GaAs: photocurrent transients [38], pump- and-probe reflectivity [39], absorption [40], timeresolved luminescence [26], optical pump-THz radiation probe [41], and analysis of $\mathrm{THz}$ emission from LTG GaAs photoswitch antennae [42]. Contribution of arsenic precipitates present in annealed LTG GaAs in the excess carrier recombination was strongly debated [18, 10]. Eventually it seems that the arsenic antisite defect itself explains the experimental observations [43], and arsenic precipitates do not play an important role in either electron trapping or electron transport. Important were the studies of LTG GaAs doped with Be impurities [29, 44, 45]. The model of this material clarified the role of arsenic antisite for the carrier recombination as well as importance of gallium vacancies for the LTG GaAs performance.

A few years after beginning of the LTG GaAs research boom, an alternative way of obtaining highly non stoichiometric As-rich GaAs by means of implantation with high energy As ions was tried $[21,46]$. It was also a subject of our common studies with A. Krotkus [21, 47-53]. The material showed carrier recombination characteristics resembling those of LTG GaAs [21]. The electron trapping time changed from a few tens of femtoseconds for as-implanted material to picoseconds after annealing at $600^{\circ} \mathrm{C}$. Implanted semi-insulating wafers with $2 \mathrm{MeV}$ ions had the maximum energy deposition density at a depth of about $0.7 \mu \mathrm{m}$, so even this size was similar to the typical thickness of the LTG GaAs epilayer. Surprisingly, it was found that implantation with other heavy ions, Ga [47], Si or O [49], produces the material of similar properties, with a high concentration of the arsenic antisite defect in the implanted layer. The proposed explanation of such behaviour connected creation of arsenic antisite defects with the mass difference of $\mathrm{Ga}$ and As. Gallium atoms, being lighter, could recoil deeper than arsenic, so the surface layer was arsenic rich and post-implantation annealing resulted in a high concentration of arsenic antisites.

The highly defected semiconductors, especially those based on LTG GaAs, are basic materials in different devices, some of them mentioned before. The review [34] covers a whole variety of methods of growth and processing leading to semi-insulating semiconductors and their heterostructures. It also presents devices in which semi-insulating layers are part of their construction necessary for 
insulation, and ultrafast devices whose performance relies on an ultrashort lifetime of excess carriers. More recent reviews of semiconductor materials for ultrafast optoelectronic devices are a subject of other publications [28, 35].

\section{Fast carrier recombination in active layers of organic solar cells}

Time-resolved luminescence and light-induced EPR are able to directly trace photo-induced charge transfer in organic solar cells [54]. The exemplary data for materials of the solar cell active layer which exhibits an efficient charge transfer are presented in Fig. 5. Figure 5 shows a temporal evolution of the luminescence spectra for $\mathrm{P} 3 \mathrm{HT}$ polymer, $\mathrm{PC}_{61} \mathrm{BM}$ fullerene, and their mixture. Efficient charge transfer is possible in this case because the energy position of HOMO and LUMO levels of $\mathrm{P} 3 \mathrm{HT}$ is higher than the corresponding ones of $\mathrm{PC}_{61} \mathrm{BM}$ fullerene [55]. The pure polymer luminescence is characterized by the lifetime of the order of a nanosecond (Fig. 5(a)). A similar lifetime is observed for the fullerene luminescence as well (Fig. 5(b)). However, for the P3HT:PC ${ }_{61} \mathrm{BM}$ mixture a significant quenching (about two orders of magnitude) of both the polymer and fullerene luminescence can be seen. Moreover, the luminescence decay time is shorter compared to that of the pure materials. This behaviour is attributed to the efficient charge transfer: light-induced excitons in $\mathrm{P} 3 \mathrm{HT}$ and $\mathrm{PC}_{61} \mathrm{BM}$, after reaching the interface of two materials through diffusion, create charge transfer excitons, with charges in different materials: holes in $\mathrm{P} 3 \mathrm{HT}$ and electrons in $\mathrm{PC}_{61} \mathrm{BM}$, and then separation of the charges may occur.

Since the photo-induced charge transfer and electron-hole separation are accompanied by the creation of two paramagnetic species, a positive and a negative polaron in $\mathrm{P} 3 \mathrm{HT}$ and $\mathrm{PC}_{61} \mathrm{BM}$, respectively, each with spin $S=1 / 2$, they can be directly detected by light-induced EPR spectroscopy. As can be seen from Fig. 6, no EPR signal of the P3HT: $\mathrm{PC}_{61} \mathrm{BM}$ blend under dark conditions is observed, whereas under illumination two lines corresponding to the polarons can be detected.

The analogous quenching of luminescence and shortening of its lifetime can be observed for the perovskite when its layer is in a direct contact
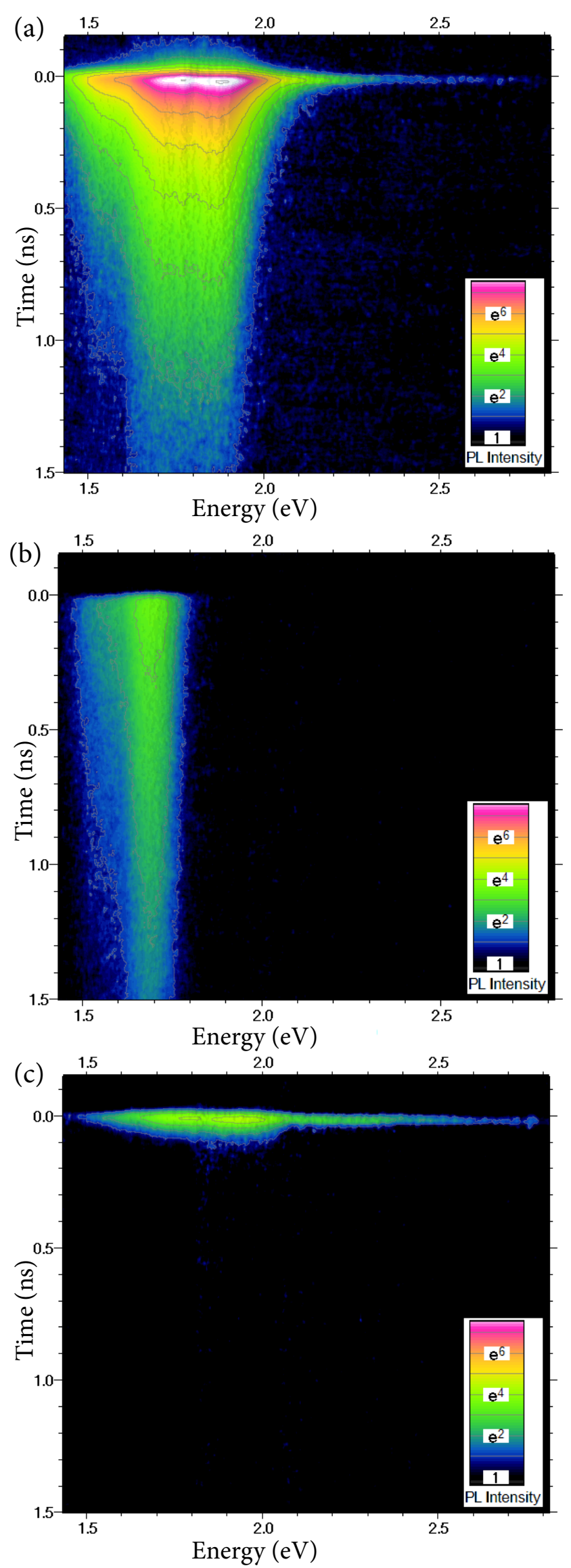

Fig. 5. Time-resolved luminescence for (a) P3HT polymer, (b) $\mathrm{PC}_{61} \mathrm{BM}$ fullerene, and (c) their mixture. 


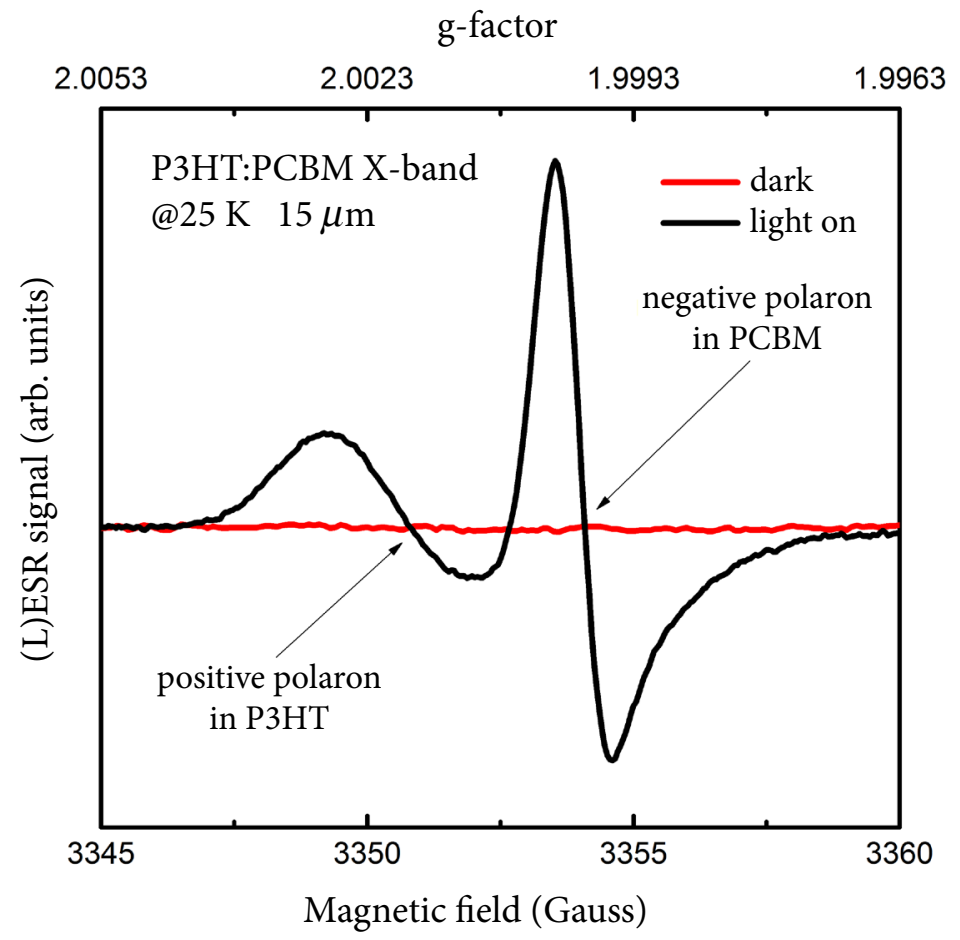

Fig. 6. EPR (red online or grey curve) and light-induced EPR (black curve) spectra of the P3HT: $\mathrm{PC}_{61} \mathrm{BM}$ blend recorded at $25 \mathrm{~K}$ with microwave power of $1.5 \mu \mathrm{W}$. with the $\mathrm{PC}_{71} \mathrm{BM}$ fullerene layer. Such structure is realized in perovskite solar cells. Figure $\mathbb{\nabla}(\mathrm{a})$ presents the strong luminescence of the pure $\mathrm{CH}_{3} \mathrm{NH}_{3} \mathrm{PbI}_{3-x} \mathrm{Cl}_{x}$ perovskite layer. Its intensity is substantially quenched and lifetime shortened for the perovskite/fullerene layered structure (Fig. B(b)). Shortening of the lifetime in a perovskite solar cell is, however, less pronounced than in a P3HT cell since the latter one is in a form of bulk heterojunction (P3HT:PCBM), and therefore the necessary diffusion length of excitons/carriers is much shorter than in the layered perovskite structure.

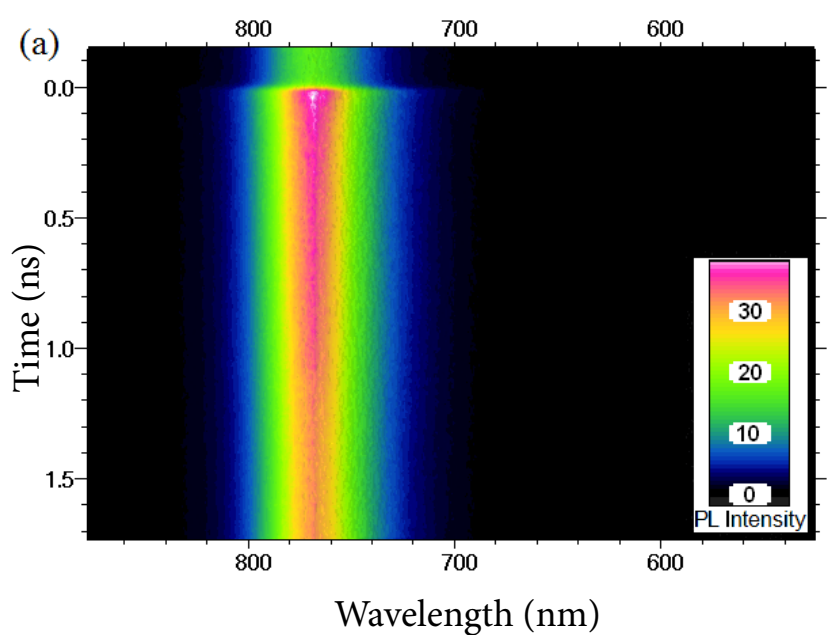

\section{Conclusions}

Radiative recombination in semiconductor materials, its intensity and lifetime are decisive for the possibility of applying these materials in a particular type of optoelectronic devices. Nonradiative processes, mostly due to the ShockleyRead-Hall recombination, are harmful in materials intended as light sources, photo-detectors and solar cells, but they are beneficial in materials used for ultrafast optoelectronics applications. Semiconductor defects and impurities are responsible for this non-radiative recombination. Depending

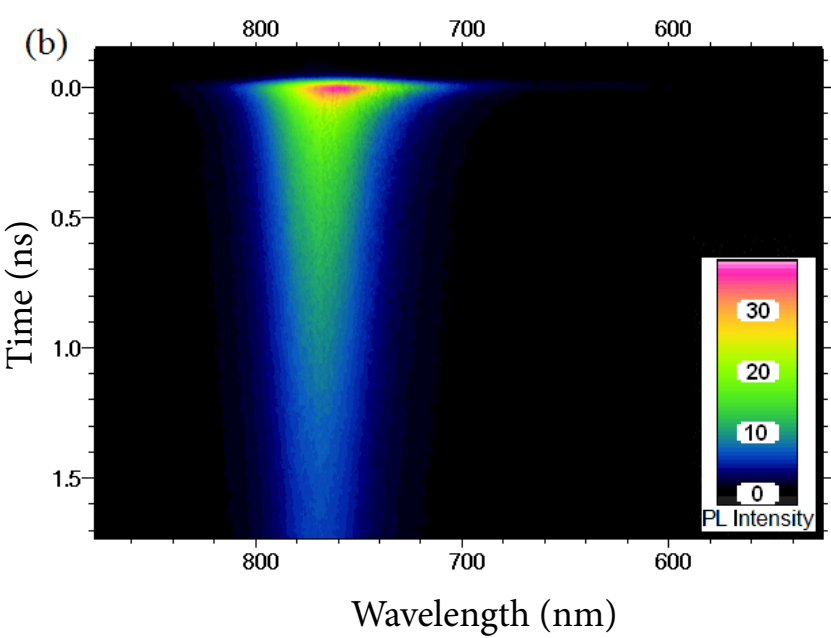

Fig. 7. Time-resolved luminescence for (a) $\mathrm{CH}_{3} \mathrm{NH}_{3} \mathrm{PbI}_{3-x} \mathrm{Cl}_{x}$ perovskite and (b) $\mathrm{CH}_{3} \mathrm{NH}_{3} \mathrm{PbI}_{3-x} \mathrm{Cl}_{x}$ perovskite/ $\mathrm{PC}_{71} \mathrm{BM}$ fullerene layered structure. 
on defect or impurity concentration and the value of its carrier capture cross-section, it is possible to control the rate of non-radiative processes, and thus also the time of decay and the intensity of luminesce. By changing defect concentration the luminescence lifetime can be shortened from nanoseconds down to subpicosecond values. Luminescence measurements, and in particular time-resolved luminescence, provide an extremely useful tool for studying recombination processes. Time-resolved luminescence is likewise very helpful for studying charge transfer processes, crucial for the efficiency of solar cells. The charge transfer process can be also traced by the photo-induced EPR technique.

\section{Acknowledgements}

The research was financed in part by the National Centre for Research and Development (Poland) under the Project TECHMATSTRATEG1/347431/14/ NCBR/2018.

\section{References}

[1] D. Curie, Luminescence in Crystals (Wiley, New York, 1963).

[2] Y.P. Varshni, G.E.J. Garlick, and A.E. Yunovich, Radiative Recombination in Semiconductors (Nauka, Moscow, 1972) [in Russian].

[3] V.L. Bonch-Bruevich and S.G. Kalashnikov, The Physics of Semiconductors (Nauka, Moscow, 1990) [in Russian].

[4] H. Spanggaard and F.C. Krebs, A brief history of the development of organic and polymeric photovoltaics, Sol. Energy Mater. Sol. Cells 83, 125146 (2004).

[5] C. Deibel and V. Dyakonov, Polymer-fullerene bulk heterojunction solar cells, Rep. Prog. Phys. 73, 96401 (2010).

[6] J. Behrends, A. Sperlich, A. Schnegg, T. Biskup, C. Teutloff, K. Lips, V Dyakonov, and R. Bittl, Direct detection of photoinduced charge transfer complexes in polymer fullerene blends, Phys. Rev. B 85, 125206 (2012).

[7] Research Cell Efficiency Records, the latest chart (NREL), https://www.nrel.gov/ncpv/

[8] K. Galkowski, A. Mitioglu, A. Miyata, P. Plochocka, O. Portugall, G.E. Eperon, J.T.-W. Wang,
T. Stergiopoulos, S.D. Stranks, H. Snaith, and R.J. Nicholas, Determination of the exciton binding energy and effective masses for methylammonium and formamidinium lead tri-halide perovskite semiconductors, Energy Environ. Sci. 9, 962-970 (2016).

[9] B.I. Shklovskii and A.L. Efros, Electronic Properties of Doped Semiconductors (Springer-Verlag, Berlin, Heidelberg, New York, Tokyo, 1984).

[10]M. Kaminska and E.R. Weber, Low temperature GaAs: electrical and optical properties, Mater. Sci. Forum 83-87, 1033-1044 (1992).

[11]H. Rohdin, M.W. Muller, and C.M. Wolfe, A model of $\mathrm{Cr}$ in GaAs, J. Phys. Chem. Solids 44, 1049-1057 (1983).

[12]G.M. Martin, A. Mitonneau, and A. Mircea, Electron traps in bulk and epitaxial GaAs crystals, Electron. Lett. 13, 191-193 (1997).

[13]E.R. Weber, H. Ennen, U. Kaufmann, J. Windscheif, J. Schneider, and T. Wosinski, Identification of AsGa antisites in plastically deformed GaAs, J. Appl. Phys. 53, 6140-6143 (1982).

[14]M. Kaminska, M. Skowronski, J. Lagowski, J.M. Parsey, and H.C. Gatos, Intracenter transitions in the dominant deep level (EL2) in GaAs, Appl. Phys. Lett. 43, 302-304 (1983).

[15]M. Kaminska, M. Skowronski, and W. Kuszko, Identification of the $0.82-\mathrm{eV}$ electron trap, EL2 in GaAs, as an isolated antisite arsenic defect, Phys. Rev. Lett. 55, 2204-2207 (1985).

[16]F.W. Smith, A.R. Calawa, C.-L. Chen, M.J. Manfra, and L.J. Mahoney, New MBE buffer used to eliminate backgating in GaAs MESFETs, IEEE Electron Device Lett. 9, 77-80 (1988).

[17] M. Kaminska, Z. Liliental-Weber, E.R. Weber, T. George, J.B. Kortright, F.W. Smith, B.-Y. Tsaur, and A.R. Calawa, Structural properties of Asrich GaAs grown by molecular beam epitaxy at low temperatures, Appl. Phys. Lett. 54, 18811883 (1989).

[18]A.C. Warren, J.M. Woodall, J.L. Freeouf, D. Grischkowsky, D.T. McInturff, M.R. Melloch, and N. Otsuka, Arsenic precipitates and the semiinsulating properties of GaAs buffer layers grown by low-temperature molecular beam epitaxy, Appl. Phys. Lett. 57, 1331-1333 (1990). 
[19]K. Bertulis, A. Krotkus, G. Aleksejenko, V. Pacebutas, R. Adomavicius, G. Molis, and S. Marcinkevicius, GaBiAs: A material for optoelectronic terahertz devices, Appl. Phys. Lett. 88, 201112 (2006).

[20]R. Adomavicius, A. Urbanowicz, G. Molis, A. Krotkus, and E. Satkovskis, Terahertz emission from $\mathrm{p}$-InAs due to the instantaneous polarization, Appl. Phys. Lett. 85, 2463-2465 (2004).

[21]A. Krotkus, S. Marcinkevicius, J. Jasinski, M. Kaminska, H.H. Tan, and C. Jagadish, Picosecond carrier lifetime in GaAs implanted with highdoses of As ions - an alternative material to lowtemperature GaAs for optoelectronic applications, Appl. Phys. Lett. 66, 3304-3306 (1995).

[22]A. Krotkus, Semiconductors for terahertz photonics applications, J. Phys. D 27, 273001 (2010).

[23]J.F. Scott, H.J. Fan, S. Kawasaki, J. Banys, M. Ivanov, A. Krotkus, J. Macutkevic, R. Blinc, V.V. Laguta, P. Cevc, J.S. Liu, and A.L. Kholkin, Terahertz emission from tubular $\mathrm{Pb}(\mathrm{Zr}, \mathrm{Ti}) \mathrm{O}_{3}$ nanostructures, Nano Lett. 8, 4404-4409 (2008).

[24]R. Adomavicius, G. Molis, A. Krotkus, and V. Sirutkaitis, Spectral dependencies of terahertz emission from InAs and InSb, Appl. Phys. Lett. 87, 261101 (2005).

[25] V.L. Malevich, R. Adomavicius, and A. Krotkus, $\mathrm{THz}$ emission from semiconductor surfaces, C.R. Phys. 9, 130-141 (2008).

[26]A. Krotkus, R. Viselga, K. Bertulis, V. Jasutis, S. Marcinkevicius, and U. Olin, Subpicosecond carrier lifetimes in GaAs grown by molecularbeam epitaxy at low substrate-temperature, Appl. Phys. Lett. 66, 1939-1941 (1995).

[27]A. Krotkus, K. Bertulis, L. Dapkus, U. Olin, and S. Marcinkevicius, Ultrafast carrier trapping in Be-doped low-temperature-grown GaAs, Appl. Phys. Lett. 75, 3336-3338 (1999).

[28]A. Krotkus and J.L. Coutaz, Non-stoichiometric semiconductor materials for terahertz optoelectronics applications, Sem. Sci. Technol. 20, S142S150 (2005).

[29]A. Krotkus, K. Bertulis, M. Kaminska, K. Korona, A. Wolos, J. Siegert, S. Marcinkevicius, J.F. Roux, and J.L. Coutaz, Be-doped low-temperaturegrown GaAs material for optoelectronic switches, IEE Proc. Optoelectron. 149, 111-115 (2002).
[30]K.P. Korona, A. Wysmolek, M. Kamińska, A. Twardowski, M. Piersa, M. Palczewska, G. Strzelecka, A. Hruban, J. Kuhl, R. Adomavicius, and A. Krotkus, Manganese as a fast charge carrier trapping center in InP, Physica B 382, 220-228 (2006).

[31]S. Gupta, M.Y. Frankel, J.A. Valdmanis, J.F. Whitaker, G.A. Mourou, F.W. Smith, and A.R. Calawa, Subpicosecond carrier lifetime in GaAs grown by molecular beam epitaxy at low temperatures, Appl. Phys. Lett. 59, 3276-3278 (1991).

[32]E.S. Harmon, M.R. Melloch, J.M. Woodall, D.D. Nolte, N. Otsuka, and C.L. Chang, Carrier lifetime versus anneal in low temperature growth GaAs, Appl. Phys. Lett. 63, 2248-2250 (1993).

[33]J.F. Whitaker, Optoelectronic applications of LTMBE III-V materials, Mater. Sci. Eng. B 22, 61-67 (1993).

[34]D.D. Nolte, Semi-insulating semiconductor heterostructures: Optoelectronic properties and applications, J. Appl. Phys. 85, 6259-6289 (1999).

[35]A. Krotkus, K. Bertulis, R. Adomavicius, V. Pacebutas, and A. Geizutis, Semiconductor materials for ultrafast optoelectronic applications, Lith. J. Phys. 49, 359-372 (2009).

[36]K.P. Korona, J. Muszalski, M. Kaminska, and E.R. Weber, Deep defects in low-temperature GaAs, Acta Phys. Pol. A 82, 821-824 (1992).

[37]S. Marcinkevicius, A. Krotkus, R. Viselga, U. Olin, and C. Jagadish, Non-thermal photoexcited electron distributions in non-stoichiometric GaAs, Semicond. Sci. Technol. 12, 396-400 (1997).

[38]F.W. Smith, H.Q. Le, V. Diaduk, M.A. Hollis, A.R. Calawa, S. Gupta, M. Frankel, D.R. Dykaar, G.A. Mourou, and T.Y. Hsiang, Picosecond GaAs based photoconductive optoelectronic detectors, Appl. Phys. Lett. 54, 890-892 (1989).

[39]J.F. Roux, J.L. Coutaz, and A. Krotkus, Timeresolved reflectivity characterization of polycrystalline low-temperature-grown GaAs, Appl. Phys. Lett. 74, 2462-2464 (1999).

[40] U. Siegner, R. Fluck, G. Zhang, and U. Keller, Ultrafast high-intensity nonlinear absorption dynamics in low-temperature grown gallium arsenide, Appl. Phys. Lett. 69, 2566-2568 (1996). 
[41]S.S. Prabhu, S.E. Ralph, M.R. Melloch, and E.S. Harmon, Carrier dynamics of low-temperature-grown GaAs observed via $\mathrm{THz}$ spectroscopy, Appl. Phys. Lett. 70, 2419-2421 (1997).

[42]H. Nemec, A. Pashkin, P. Kuzel, M. Khazan, S. Schnull, and I. Wilke, Carrier dynamics in lowtemperature grown GaAs studied by terahertz emission spectroscopy, J. Appl. Phys. 90, 13031306 (2001).

[43]M. Kaminska and E.R. Weber, The Physics of Semiconductors, eds. E.M. Anastasskis and J.D. Joannopoulos (World Scientific, Singapore, 1990) p. 473.

[44]A.J. Lochtefeld, M.R. Melloch, J.C.P. Chang, and E.S. Harmon, The role of point defects and arsenic precipitates in carrier trapping and recombination in low-temperature grown GaAs, J. Appl. Phys. 64, 1465-1467 (1996).

[45]M. Haiml, U. Siegner, F. Morier-Genoud, U. Keller, M. Luysberg, P. Specht, and E.R. Weber, Femtosecond response times and high optical nonlinearity in beryllium-doped low-temperature grown GaAs, Appl. Phys. Lett. 74, 1269-1271 (1999).

[46]A. Claverie, F. Namavar, and Z. Liliental-Weber, Formation of As precipitates in GaAs by ion implantation and thermal annealing, Appl. Phys. Lett. 62, 1271-1273 (1993).

[47]C. Jagadish, H.H. Tan, J. Jasinski, M. Kaminska, M. Palczewska, A. Krotkus, and S. Marcinkevicius, High resistivity and picosecond carrier lifetime of GaAs implanted with $\mathrm{MeV}$ Ga ions at high fluences, Appl. Phys. Lett. 67, 1724-1726 (1995).

[48]J. Jasinski, A. Kurpiewski, K. Korona, M. Kaminska, M. Palczewska, A. Krotkus, S. Marcinkievicius, Z. Liliental-Weber, H.H. Tan, and C. Jagadish, Role of arsenic antisite defects in nonstoichiometric gallium arsenide, Acta Phys. Pol. A 88, 747-750 (1995).
[49]C. Jagadish, H.H. Tan, A. Krotkus, S. Marcinkevicius, K.P. Korona, and M. Kaminska, Ultrafast carrier trapping in high energy ion implanted gallium arsenide, Appl. Phys. Lett. 68, 2225-2227 (1996).

[50]H.H. Tan, C. Jagadish, K.P. Korona, J. Jasinski, M. Kaminska, R. Viselga, S. Marcinkevicius, and A. Krotkus, Ion-implanted GaAs for subpicosecond optoelectronic devices, IEEE J. Sel. Top. Quantum Electron. 2, 636-642 (1996).

[51]K.P. Korona, J. Jasinski, A. Kurpiewski, M. Kaminska, C. Jagadish, H.H. Tan, A. Krotkus, and S. Marcinkevicius, Ultrafast carrier trapping and high resistivity of $\mathrm{MeV}$ energy ion implanted GaAs, Acta Phys. Pol. A 90, 851-854 (1996).

[52]A. Krotkus, S. Marcinkevicius, C. Jagadish, and M. Kaminska, Femtosecond electron relaxation in non-stoichiometric GaAs and InGaAs, J. Lumin. 66-67, 455-461 (1996).

[53]S. Marcinkevičius, C. Jagadish, H.H. Tan, M. Kaminska, K. Korona, R. Adomavičius, and A. Krotkus, Influence of annealing on carrier dynamics in As ion-implanted epitaxially lifted-on GaAs layers, Appl. Phys. Lett. 76, 1306-1308 (2000).

[54]S. Grankowska Ciechanowicz, K.P. Korona, A. Wolos, A. Drabinska, A. Iwan, I. Tazbir, J. Wojtkiewicz, and M. Kaminska, Toward better efficiency of air-stable polyazomethine-based organic solar cells using time-resolved photoluminescence and light-induced electron spin resonance as verification methods, J. Phys. Chem. C 120, 11415-11425 (2016).

[55]J. Wojtkiewicz, A. Iwan, M. Pilch, B. Boharewicz, K. Wojcik, I. Tazbir, and M. Kaminska, Towards designing polymers for photovoltaic applications: A DFT and experimental study of polyazomethines with various chemical structures, Spectrochim. Acta A 181, 208-217 (2017).

\title{
RADIACINĖ REKOMBINACIJA IR KITI SUSIJE SU PERTEKLINIAIS KRŪVININKAIS VYKSMAI, LEMIANTYS ELEKTRONINIŲ PRIETAISŲ EFEKTYVUMA
}

\author{
A. Wincukiewicz, W. Mech, S. Grankowska, A. Wołoś, A. Drabińska, T. Słupiński, K.P. Korona, \\ M. Kamińska
}

